\title{
El sentido del texto jurídico y la interpretación ética en el Derecho
}

\section{The meaning of the text and interpretation legal ethics in Law}

Carlos Mario Dávila S.

Magíster en Derecho comparado Universidad de La Sorbona (París I). Francia.

Catedrático Universitario.

Recibido: 27/04/2012 Aprobado: 18/09/2012

carmdavs@gmail.com

\section{RESUMEN}

El presente artículo, se presenta en dos momentos. En primer lugar, aborda el tema de la interpretación y los sentidos del texto jurídico, demostrando que estos no poseen una verdad en sí mismos y contario a lo que se cree, señalan varios e infinitos significados. En segundo lugar, propone un concepto de la interpretación ética en derecho de los textos jurídicos, los cuales son fuente esencial del conocimiento para todos los juristas, y a su vez demuestra cómo esta interpretación ética puede ser utilizada en la aplicación de derechos fundamentales. Todo lo anterior teniendo en cuenta que en el campo de los estudios jurídicos, el margen de la interpretación es limitada y por tanto juega un papel importante el talento que debe poseer el individuo al momento realizar este ejercicio.

\section{PALABRAS CLAVE}

Interpretar, texto jurídico, ética, Derecho.

\section{ABSTRACT}

The following article is presented in two parts. First, it addresses the issue of the interpretation and the senses of the legal text, proving that this don't have a truth in themselves and contrary to what is believed, they point to various and infinite meanings. Seconds, proposes a concept of ethics in law interpretation of legal texts. Which are essential source of knowledge for all lawyers, In turn, this interpretation demonstrates how ethics can be used in the application of fundamental rights. All the above taking into account that in the field of law studies, the interpretation margin is limited and therefore plays an important role the talent that everyone must possess when performing this exercise.

\section{KEYWORDS}

Interpret, legal texts, ethic, law. 


\section{INTRODUCCIÓN}

Durante toda la historia de la humanidad se ha buscado establecer el sentido de la cosa, el ser. Empero, esto no ha sido posible hasta el presente, $y$, por consiguiente, cuando alguien decía esto 'es', no hacía otra cosa que decir esto 'significa'.' En lo que concierne a los textos, cualquiera sea su tipo, se puede emitir una afirmación parecida. Primero con la Biblia, ${ }^{2}$ después con los textos jurídicos y filosóficos; $y$, finalmente, con toda suerte de escritos. Ahora bien, pensadores han propuesto diferentes métodos en diversas épocas, pero, esos métodos no han sido suficientes o llevaron a la misma conclusión, a saber, la verdad, la cosa en sí, el sentido fijo y único de un texto, no pueden ser identificados. ${ }^{3}$

A lo largo de estas líneas se intentará mostrar que los textos jurídicos no poseen una verdad en ellos mismos y que no habría tan sólo un sentido fijo y único a identificar en un escrito jurídico, sino al contrario: que existen varios e infinitos significados como en el plumaje tornasolado de un pavo real (Borges, 2005, p.9). Ahora bien, aunque se pueda interpretar de diferentes maneras un texto y que cada quien pueda dar a un escrito un sentido distinto, es necesario hacer algunas precisiones sobre el carácter ético de la interpretación. En efecto, puesto que no hay un solo sentido en un texto y que los libros no significan nada en sí (Borges, 1995, p.90), "el objetivo de la interpretación no es entonces de reconstituir con una absoluta certitud el pensamiento auténtico del autor sino de prestarle el mejor pensamiento posible en función de lo que él dice" ${ }^{\mu 4}$, adoptado una postura ética ${ }^{5}$ de respeto del autor, del texto y del contexto. La interpretación ética en el derecho deviene entonces imperiosa, al momento de adjudicar una significación, pues uno puede no solamente equivocarse sobre el nombre sino también sobre el concepto $y$, en consecuencia, conllevar a un malentendido. ${ }^{6}$

1.- Friedrich NIETZSCHE, La volonté de puissance I, trad. por Geneviève BIANQUIS, París, Gallimard, 1995 [1935], p. 44: “Pero cada vez, hasta presente, que alguien declaró: 'esto es', hubo una época ulterior más afinada para descubrir que estas palabras no tenían sino un sólo sentido posible: 'esto significa'”. ("Mais chaque fois, jusqu'à présent, que l'on a déclaré : 'Cela est', il s'est trouvé une époque ultérieure plus affinée pour découvrir que ces mots n'avaient qu'un seul sens possible : 'Cela signifie'"). [Las comillas son del autor]. Ver en castellano, Federico NIETZSCHE, La Voluntad de poder, trad. por Aníbal Froufe, Madrid, Edaf, 2006.

2.-Ver Umberto ECO, "Interprétation et histoire", en Umberto ECO y al., Interprétation et surinterprétation, trad. por Jean-Pierre COMETTI, Paris, P.U.F., 2002 [1992], p. 3: "La interpretación evidentemente no es una actividad inventada por los teóricos de la literatura en el siglo XX [...]. La fuente mayor fue la tarea que buscó establecer el significado de la palabra de Dios". ("L'interprétation n'est évidemment pas une activité inventée par les théoriciens de la littérature au XXe siècle [...]. La source majeure en fut la tache [...] visant à établir la signification de la parole de Dieu"). Ver en castellano, Umberto ECO y al., Interpretación y sobreinterpretación, trad. por Juan Gabriel López, Madrid, Cambridge UP, 2002.

3.- Por su parte, PLATÓN concluye su diálogo el Cratylo sosteniendo que la cosa en sí existe pero que a causa del movimiento ella no puede ser aprehendida, CEuvresComplètes, vol. I, trad. por Léon ROBIN, París, Gallimard, 1950, pp. 688-91: "[Sócrates]: ¿debemos decir que existe un Bello, un Bueno en sí, y lo mismo para cada una de las realidades individualmente? ¿O bien negarlo? - Cratilo: No, pero claro que existe; [...] [Sócrates]: ¿no es una necesidad que, en el tiempo en el cual estamos hablando de ello, deviene [lo Bello, lo Bueno] tan pronto, otro sí, que a nuestra espalda se evade, que no se comporta más de la misma manera? -Cratilo: Necesariamente." ("[Socrate] : devons-nous dire qu'il existe un Beau, un Bon en soi, et de même pour chacune des réalités individuellement ? Ou bien de le nier? - Cratyle : Non, mais qu'il existe ; [...] (Socrate) : n'est-ce pas une nécessité que, dans le temps même où nous en parlons, tout aussitôt il [le Beau, le Bon] devienne un autre soi, qu'à notre insu il s'évade, qu'il ne se comporte plus de même ? - Cratyle : Nécessairement"). Ver en castellano, PLATÓN, Cratilo en Diálogos, trad. por Varios, Madrid, Gredos, 2011. Para el WITTGENSTEIN del Tractatus el problema residía en el lenguaje, ver Ludwig WITTGENSTEIN, Tractatus lógico-philosophicus, Londres, Routledge\&Kegan Paul, 1951 [1922] "La mayoría de las proposiciones y cuestiones de los filósofos vienen de nuestra incomprensión de la lógica de la lengua." ("Most questions and propositions of the philosophers result from the fact that we do not understand the logic of our language.")

4.-Ariel SUHAMY, "Sens et vérité: L'interprétation selon SPINOZA" en L'interprétation, bajo la dir. de Patrick WOTLING, Paris, Vrin, 2010, p. 61.

5.-Para una profundización sobre la noción de 'ética' ver Felipe PRIETO, "La ética en la antigüedad", en Filosofía del derecho, bajo ladir. de Miguel RUJANA QUINTERO, Bogotá, Ibáñez, 1999, pp. 3-24.

6.- Ver en este sentido Stéphane MARCHAND, "Saint Augustin et l'éthique de l'interprétation", en L'interprétation, bajo la dir. de Patrick WOTLING, Paris, Vrin, 2010, p. 34: "La interpretación es necesaria porque, para nosotros y entre nosotros, un malentendido siempre es posible". ("L'interprétation est nécessaire parce que, pour nous et entre nous, un malentendu est toujours possible"). 
De esta manera, se abordará en una primera parte la cuestión de la interpretación y los sentidos del texto jurídico, para luego, proponer un concepto de la interpretación ética en derecho, pues no es cierto que todo se vale en términos de interpretación y que uno puede dar cualquier sentido a un escrito jurídico, comprendida una norma.

\section{LA INTERPRETACIÓN JURÍDICA Y LOS SIGNIFICADOS DEL TEXTOENEL DERECHO}

¿Qué es interpretar? Es la pregunta que alguna vez nos hacemos, pero a la cual los juristas no han intentado ciertamente responder, al menos en los cursos de derecho. ${ }^{7}$ Sin embargo, el jurista se enfrenta todos los días a textos, palabras, enunciados, normas jurídicas y decisiones de jurisprudencia. Es por ello, que es menester afirmar de entrada que la interpretación es ante todo un arte, ${ }^{8}$ así como el derecho también lo es, ${ }^{9}$ es por ello entonces, que el talento del individuo que interpreta se ve reflejado en la interpretación que realiza.

En general, cuando se va a la facultad de jurisprudencia, leyes o derecho, se va para aprender el 'canon' o la interpretación correcta de la norma jurídica. De esta manera, el discente aprende lo que el profesor dice que la norma 'es'. Lo mismo acontece en la escuela primaria o secundaria, el alumno aprende lo que el instructor aprendió que la cosa 'era'. Por consiguiente, se evalúa en función de los conocimientos dados por la persona encargada de enseñar, si el estudiante responde en concordancia con lo que el profesor ha enseñado, se dirá que éste habrá aprendido bien la materia. Ahora bien, aquí se está en presencia de una 'verdad' pero en función de alguna cosa, en el ejemplo, el referente de lo que será verdadero o erróneo, es el conocimiento transmitido por el enseñante y los libros en los cuales se basó. Entonces, como la 'verdad' que aquí se presenta existe pero en función de alguna cosa, y no en sí misma, como absoluta y universal, se debe remplazar la palabra 'verdad' por certitud 0 verosimilitud.

Los textos jurídicos están afectados de la misma situación, además de que uno no puede reconocer en realidad la intención del autor. ${ }^{10}$ Por otra parte, un texto puro no existe, de la misma manera que "[u]n autor puro no existe en ningún lado". ${ }^{11}$ De contera, un escrito puede contener una cierta cantidad de enunciados que pueden ser catalogados de 'verdaderos', pero esto no será que en función de algo. Así, si el enunciado siguiente "[l]a Constitución Política podrá ser reformada por el Congreso, por una Asamblea constituyente o por el pueblo mediante referendo" es calificado de verdadero, es porque existe un texto que confirma mi afirmación, en este caso la Constitución en su artículo 374, pero no porque esto sea una verdad en sí. Y para hacer esto fue necesario haber interpretado y haber adjudicado un significado al texto en cuestión. ${ }^{12}$

Además, para poder afirmar que "[l]a Constitución Política podrá ser reformada por el Congreso, por una Asamblea constituyente o por el pueblo mediante

7.-Para una clasificación de los métodos dominantes en la interpretación jurídica ver Riccardo GUASTINI, "Interprétation et description de normes" en Interprétation et droit, bajo la dir. de Paul AMSELEK, Bruselas, Bruylant, 1995, pp. 89-101.

8.-Ver en este sentido Christian BERNER, “'Interpréter est un art'. Les grandes lignes de I'herméneutique de Schleiermacher", en L'interprétation, bajo la dir. de Patrick WOTLING, París, Vrin, 2010, pp. 63-84: "La hermenéutica es el 'arte de comprender'” ("L'herméneutique est 'art de comprendre'") [Las comillas son delautor].

9.-Ver Marcel MAUSS, La religion et les origines du droit pénal d'après un livre récent, en CEuvres, vol. II, París, Éditions de Minuit, 2001 [1896], p. 652: "Pero el derecho no es una ciencia, es un arte [y una técnica]". ("Mais le droit n'est pas une science, c'est un art"). Ver en castellano, Marcel MAUSS, Obras, 3 vols., trad. por Juan Antonio Matesanz, Barcelona, Barral, 1970-72.

10.- Para el Profesor ECO existe la intención del autor, la intención del lector, pero también la intención del texto, ver op. cit.,nota 2, p. 23. 11.- Christine BROOKE-ROSE, "Histoire palimpseste", en Umberto ECO y al.,Interprétation et surinterprétation, París, P.U.F., 2002 [1992], p. 123. Ver en castellano, Umberto ECO y al., Interpretación y sobreinterpretación, trad. por Juan Gabriel López, Madrid, Cambridge UP, 2002, pp. 143-58.

12.- Ver Michel TROPER, “Interprétation”, en Dictionnaire de la culture juridique, bajo ladir. de Denis ALLAND y Stéphane RIALS, París, P.U.F., 2003, p. 844: "No hay significación antes de la interpretación auténtica". ("[I]I n'y a pas de signification avant l'interprétation authentique"). 
referendo", es necesario convenir sobre todas las palabras que conforman esta frase. ¿Qué es una Constitución Política? ¿Qué es una reforma? ¿Qué es un Congreso? ¿Qué es una Asamblea constituyente? ¿Qué es el pueblo? ¿Qué es un referendo? Son preguntas que a primera vista pueden parecer obvias pero que, sacadas de contexto pueda que no lo sean tanto. Es ahí donde la interpretación juega su papel decisivo. Aun Hans KELSEN de la indeterminación de los conceptos y por consiguiente, él sabía que no es posible adjudicar a un texto jurídico un solo y único significado. ${ }^{13}$ Pero es ahí, también, donde las escuelas primarias, secundarias y las universidades juegan su papel decisivo. En efecto, cuando se va a la escuela, se comienza a aprender y a fijar en el individuo una lengua, la cual se empezó a oír y a hablar en casa. Esta lengua tiene por fin el 'poder comunicar'. Para comunicar es necesario ponerse de acuerdo sobre ciertos aspectos. Si vemos una manzana, es necesario que cuando uno de los interlocutores pronuncie esa palabra, el otro tenga, 0 los otros, tengan en mente la misma fruta, el mismo objeto, y no otro. Uno de los objetivos de la escuela es de enseñar y fijar en el individuo la lengua de la sociedad en la cual se vive, pero también, de "aprender a los pequeños qué tipo de conjeturas fueron reconocidas como verdaderas en el pasado". ${ }^{14}$ En consecuencia, el hecho de haber seguido una instrucción nos limita en el momento mismo de la interpretación.

En los estudios superiores sucede lo mismo. En la universidad el estudiante aprende el 'canon' interpretativo y el lenguaje jurídico; cómo interpretar una norma de tal manera y no de otra. El estudiante sabe desde entonces, por ejemplo, que una revisión de la Constitución por una Ley constitucional, no es la misma cosa que una revisión por vía de referendo, y, a contrario, la gente de afuera de la escena jurídica podría pensar otra cosa. El discente está restringido entonces por otra limitación hermenéutica que es aquella de los conceptos aprendidos en la facultad. El campo interpretativo deviene entonces más estrecho. Ahora bien, ¿se podría afirmar que existe una y sólo una interpretación correcta de un texto jurídico? La respuesta es negativa. En efecto, aunque el individuo haya ido a una escuela primaria, y que se pueda afirmar que toda educación tiene algo de adoctrinamiento, ${ }^{15}$ después a un establecimiento secundario, y, finalmente, a una universidad, esto no querrá jamás decir que él haya aprendido la 'verdad' en su dominio o campo de estudios. Puesto que, de una parte, el profesor ha hecho lo mismo, y, de otra parte, aunque se haya seguido los mismos cursos que otros individuos, siempre quedará un lugar para la indeterminación, así como cuando se relee un texto la interpretación cambia con relación a la primera. ${ }^{16}$ Es por ello que, el campo interpretativo, aunque reducido, deja espacio a varias interpretaciones posibles del mismo escrito.

Volviendo al texto jurídico, si se lee otro enunciado jurídico como "Colombia es una República unitaria", se puede aprender varias cosas, pero también surgen varios interrogantes. ¿Qué quiere decir la palabra República? ¿Qué significa la palabra unitaria? Para dar respuesta a ello, es necesario comprender que existe una clasificación según la organización territorial que divide los Estados en

13.-Théorie pure du droit, trad. por Charles EISENMANN, París, Dalloz, 1962 [1960], p. 458: (No hay pura y simplemente ningún método [...] que permitiría distinguir, entre varios significados lingüísticos de una norma, uno solo, que sería el verdadero significado). ("[I]I n'y a purement et simplement aucune méthode [...] qui permettrait de distinguer, entre plusieurs significations linguistiques d'une norme, une seule, qui serait la vraie signification"). Ver en castellano, Hans KELSEN, Teoría pura del derecho, trad. por Roberto Vernengo, México D.F., Porrúa, 2009.

14.-Umberto ECO, "Réponse", en Umberto ECO y al.,Interprétation et surinterprétation, París, P.U.F., 2002 [1992],p. 134. Ver en castellano, Umberto ECO y al., Interpretación y sobreinterpretación, trad. por Juan Gabriel López, Madrid, Cambridge UP, 2002, pp. 159-72.

15.-Ver al respecto Iván ILLICH, “La Sociedad desescolarizada”, en Obras reunidas, trad. por Javier Sicilia y Valentina Borremans, Vol. I, México D.F., F.C.E., 2006.

16.- Ver al respecto Jonathan CULLER, "Défense de la surinterprétation", en Umberto ECO y al., Interprétation et surinterprétation, trad. por Jean-Pierre Cometti, París, P.U.F., 2002 [1992], p. 114. Ver en castellano, Umberto ECO y al., Interpretación y sobreinterpretación, trad. por Juan Gabriel López, Madrid, Cambridge UP, 2002, pp. 127-42. 
unitarios y federales o federados, y, luego en republicanos, aristocráticos, monárquicos, etc. Por lo tanto Colombia hace parte del primer grupo de Estados. ¿Pero este sentido estaba establecido de entrada en el texto? Forzoso es concluir que no, y que para ello fue necesario haber interpretado de antemano, adjudicar un significado a cada palabra, de acuerdo una serie de conocimientos preadquiridos por parte del intérprete antes de realizar el acto de interpretación. Es por ello que cuando algún otro individuo, sin tener esa formación previa, realiza una lectura del mismo texto, su interpretación será diferente y para muchos, quizás, errónea, de ahí los conceptos de verdad y error. Cuando alguien coincide con lo que se considera como dominante en una rama del conocimiento se dirá que está en verdad. ¿Por qué, entonces, se puede tener dos 0 más interpretaciones sobre un mismo escrito? Sencillamente porque cada quien llega con su propio bagaje conceptual el cual determina su concepción del mundo (Eco, 2002, p.140). Se ve entonces que el campo interpretativo deja lugar a divergencias pues "la significación no es algo de universal que sería compartido por todos los individuos (Berner, 2010, p.67).

Se es sin embargo consciente de que si bien existe una posibilidad extendida de interpretar un texto jurídico, "hay en alguna parte normas que permiten limitar la interpretación" (Eco, 2002, p.36), de ahí que uno no pueda atribuir cualquier interpretación a un escrito. Se vio arriba, la escuela nos limita, la universidad, la lengua en la cual estamos inmersos, así como la sociedad. Sin quererlo, nos hemos puesto de acuerdo sobre una gran cantidad de nombres y de conceptos, aunque siempre pueda quedar algo de indeterminación en estos últimos. Es por ejemplo, el caso de conceptos tan indeterminados como los derechos fundamentales, donde se dan con frecuencia interpretaciones divergentes, en ocasiones se han utilizado para autorizar algo, en otras para prohibirlo. Es por ello que en la segunda parte de este escrito se tratará de dar argumentos en vista de una interpretación ética en el derecho pues "no es verdad que todo se vale" (Eco, 2002, p.133).

\section{LAINTERPRETACIÓNÉTICAEN EL DERECHO}

El jurista debe principalmente estudiar textos. Es la fuente esencial de su conocimiento. Él interpreta esos textos, asigna significados, da su punto de vista o simplemente, expresa lo que ha comprendido. Cuando el jurista hace la interpretación de un escrito, puede suceder que éste utilice esta interpretación con un fin determinado. Por ejemplo, querrá coadyuvar un argumento, en un debate, una audiencia, un discurso, etc. Esta utilización del texto puede, a veces, ser llevada fuera de contexto, esto lleva entonces a proponer aquí una interpretación ética en derecho. Esta interpretación debe tener en cuenta el autor, su vida, su obra, el texto, el contexto y la lengua en la que está escrito.

En lo que concierne a la obra, no se debe tener en cuenta tan sólo una frase, por ejemplo, aquella que convendría más, sino que se debe ir más allá, leer el texto completo, posteriormente la obra, así como la biografía del autor. Puesto que la obra de un autor no se comprende sin estudiar también su vida, es necesario abordar una biografía confiable sobre el autor. Por otro lado, el contexto, la época en la cual vivió, su lugar de residencia, la lengua en la cual escribió, y no su traducción, deben ser tenidos en cuenta y conocidos antes de utilizar un argumento de un autor. En efecto, en materia jurídica, como en otros campos, uno se enfrenta con frecuencia a autores de los cuales no se conoce mucho, es por ello que el conocimiento del contexto (Guastini, 1995, pp.89-101), la historia en la que un texto jurídico o una norma fue producida es imperiosa antes de emitir un juicio de valor o de su trasplante o utilización en otro ordenamiento jurídico. Todo esto con el fin de realizar una interpretación concordante con lo que el autor en cuestión quiso decir, pero también, para no utilizarlo en vista de otros intereses que aquellos que el mismo escritor no habría suscrito si estuviese en vida o si leyese esa cita o interpretación que se hace de él mismo.

Esta interpretación ética puede igualmente ser utilizada en la aplicación de derechos fundamentales. En efecto, puesto que estos son 
conceptos muy indeterminados, son utilizados frecuentemente para justificar un comportamiento, y en otros, para prohibirlo, v. gr., la igualdad. En ese momento una interpretación ética se vuelve necesaria. Esta interpretación es ética en el sentido que toma en cuenta no sólo el interés individual sino también el colectivo, pero también, y lo más importante, el respeto de la naturaleza como tal, en el sentido fuerte, como esencia y fuente de la vida, y, finalmente, el respeto de la otra sociedad 0 comunidad.

Este tipo de interpretación ética fue, en efecto, uno de los elementos propuestos por San Agustín para leer la Biblia. ${ }^{17}$ Aquí se propone utilizarla para interpretar tanto textos jurídicos como escritos en general. Es bien sabido, por lo que se arguyó supra, que en ocasiones se puede hacer decir a un texto lo que uno quiere, pero hay que hacerse una pregunta: ¿qué es lo que se busca con ello? Esta es la pregunta a hacerse cuando se interpreta un texto jurídico con el fin de una utilización. Por ello, la reflexión antes de utilizar una cierta interpretación es la cuestión que impone la ética en el derecho. De ahí que sea importante pensar en el autor: ¿estaría de acuerdo con la interpretación que estoy haciendo de su escrito? en el texto: ¿son realmente las palabras y el sentido que está ahí plasmado? y en el contexto: ¿en esa cultura, sociedad y lugar se interpretaría de esta manera?

Ahora bien, puede que una cierta sobreinterpretación sea necesaria, a causa de lo cual no habría imaginación. ${ }^{18}$ En efecto, en la interpretación, por ejemplo, de textos literarios una "dosis de paranoia" ${ }^{\prime 19}$ es importante pues "[u]n hombre que no habría vivido profundamente la atracción de la 'sobre-interpretación' sería incapaz de crear los personajes y las obsesiones que animan sus novelas" ${ }^{\prime 20}$. La literatura necesita de ello, para crear, inventar y hacer ficciones sobre la vida en sociedad. Pero, en el campo de los estudios jurídicos, concretamente en la interpretación el margen es limitado, es por ello que la interpretación es tan estrecha en el derecho. Empero, esto no impide que no se pueda ensayar 0 tratar de dar otra interpretación a un texto jurídico, pero siempre y cuando se haga dentro de las fronteras de una interpretación ética, a saber, teniendo en cuenta el autor, el texto y el contexto.

\section{CONCLUSIÓN}

Se intentó mostrar que un texto jurídico puede ser interpretado de diferentes maneras, pues no hay el sentido del texto sino los sentidos del texto. ${ }^{21}$ Más adelante se argumentó con el fin de la utilización ética del texto en el derecho. También se remarcó la importancia y relevancia del talento del individuo en el momento de realizar una interpretación. La ética del intérprete juega un papel importante en el momento de adjudicar una significación a un escrito jurídico, ya sea una norma, una decisión de jurisprudencia o cualquier otro tipo de texto. El arte de interpretar demanda que la persona sea humilde pues "una interpretación se convierte en nefasta a partir del momento en el que ella pretende ser la única válida y entiende decretar de entrada y, por todo, lo que ella cree verdadero haciendo economía del sentido". ${ }^{22}$

17.- Ver MARCHAND, op. cit., nota 8, pp. 12-36

18.- ECO, op. cit., nota 16, p. 133: "Admito con Culler que aun la sobre-interpretación es fecunda". ("J'admets avec CULLER que même la surinterprétation est féconde")

19.- CULLER, op. cit., nota 18, p. 105.

20.-Id.,p. 102.

21.-Ver ECO, op. cit., nota 16, p. 130: "Acepto la idea según la cual un texto puede tener varios sentidos". ("IJ]'accepte l'idée selon laquelle un texte peut avoir de nombreux sens").

22.-SUHAMY, op. cit., nota 6, p. 55. 


\section{REFERENCIAS BIBLIOGRÁFICAS}

- Borgues, J. (1980). Siete noches. Madrid: Alianza Editorial. 2005.

- Borgues, J. (1944). Ficciones. Madrid: Alianza Editorial. 1995

- Eco, U. (1992) Interprétation et historie. Paris : Ediciones Grasset 2002.
- Berner (2010).interpréter est un art les grandes lignes de L'herméneutique de Scheleirmacher. París:Vrin.

- Guastini, R. (1995) Interpétation et descrption de normes en interpretation et droit. Bruselas. Bruylant. 